\title{
A Novel Cysteine Protease Inhibitor of Naegleria fowleri That Is Specifically Expressed during Encystation and at Mature Cysts
}

\author{
Hương Giang Lê ${ }^{1,2}$, A-Jeong Ham ${ }^{3}$, Jung-Mi Kang 1,2, Tuấn Cường Võ ${ }^{1,2}$, Haung Naw ${ }^{1,2}$, Hae-Jin Sohn ${ }^{3}$, \\ Ho-Joon Shin ${ }^{3}$ and Byoung-Kuk $\mathrm{Na}^{1,2, *}$ \\ 1 Department of Parasitology and Tropical Medicine, and Institute of Health Sciences, Gyeongsang National \\ University College of Medicine, Jinju 52727, Korea; gianglee291994@gmail.com (H.G.L.); \\ gim9951001@hanmail.net (J.-M.K.); vtcuong241@gmail.com (T.C.V.); haungnaw23@gmail.com (H.N.) \\ 2 Department of Convergence Medical Science, Gyeongsang National University, Jinju 52727, Korea \\ 3 Department of Microbiology, Ajou University College of Medicine, Suwon 16499, Korea; \\ dkwjd0521@naver.com (A.-J.H.); hj35good@ajou.ac.kr (H.-J.S.); hjshin@ajou.ac.kr (H.-J.S.) \\ * Correspondence: bkna@gnu.ac.kr; Tel.: +82-55-772-8102; Fax: +82-55-772-8109
}

\section{check for} updates

Citation: Lê, H.G.; Ham, A-J.; Kang, J.-M.; Võ, T.C.; Naw, H.; Sohn, H.-J.;

Shin, H.-J.; Na, B.-K. A Novel

Cysteine Protease Inhibitor of

Naegleria fowleri That Is Specifically

Expressed during Encystation and at Mature Cysts. Pathogens 2021, 10, 388 https://doi.org/10.3390/

pathogens 10040388

Academic Editor: Valentina

Virginia Ebani

Received: 11 March 2021

Accepted: 22 March 2021

Published: 24 March 2021

Publisher's Note: MDPI stays neutral with regard to jurisdictional claims in published maps and institutional affiliations.

Copyright: (c) 2021 by the authors. Licensee MDPI, Basel, Switzerland. This article is an open access article distributed under the terms and conditions of the Creative Commons Attribution (CC BY) license (https:// creativecommons.org/licenses/by/ $4.0 /)$

\begin{abstract}
Naegleria fowleri is a free-living amoeba that is ubiquitous in diverse natural environments It causes a fatal brain infection in humans known as primary amoebic meningoencephalitis. Despite the medical importance of the parasitic disease, there is a great lack of knowledge about the biology and pathogenicity of N. fowleri. In this study, we identified and characterized a novel cysteine protease inhibitor of $N$. fowleri (NfCPI). NfCPI is a typical cysteine protease inhibitor belonging to the cystatin family with a Gln-Val-Val-Ala-Gly (QVVAG) motif, a characteristic motif conserved in the cystatin family of proteins. Bacterially expressed recombinant NfCPI has a dimeric structure and exhibits inhibitory activity against several cysteine proteases including cathespin Bs of $N$. fowleri at a broad range of $\mathrm{pH}$ values. Expression profiles of $n f c p i$ revealed that the gene was highly expressed during encystation and cyst of the amoeba. Western blot and immunofluorescence assays also support its high level of expression in cysts. These findings collectively suggest that NfCPI may play a critical role in encystation or cyst formation of $N$. fowleri by regulating cysteine proteases that may mediate encystation or mature cyst formation of the amoeba. More comprehensive studies to investigate the roles of NfCPI in encystation and its target proteases are necessary to elucidate the regulatory mechanism and the biological significance of NfCPI.
\end{abstract}

Keywords: Naegleria fowleri; cysteine protease inhibitor; encystation; cyst

\section{Introduction}

Naegleria fowleri is a free-living amoeba that is ubiquitously distributed in diverse natural environments including freshwaters and soil [1]. However, it can infect humans and cause a rare but fatal brain infection known as primary amoebic meningoencephalitis (PAM) [2]. Once the parasite enters the human body via the nasal route, it crosses the olfactory neuroepithelia, travels to the central nervous system, and eventually reaches the brain. The clinical manifestations of $N$. fowleri infection normally appear within 2-8 days after infection, which include symptoms of fever, severe headache, nausea, chills, neck stiffness, seizures, and coma and eventually result in fatal PAM characterized by necrotizing, fulminant, and hemorrhagic meningoencephalitis $[2,3]$. The progression of PAM is rapid, and usually results in death within 14 days. Until 2018, 145 cases were reported in the United States, but the mortality rate was extremely high, at 97\% [4]. Concern for expansion of PAM associated with climate changes has also increased in recent years [5]. Cases of PAM induced by $N$. fowleri infection have also been reported in diverse global areas, including Asia, Africa, Europe, and South America [6].

Pathogenesis of PAM has not been fully understood, but it has been proposed that $N$. fowleri may induce cell death and an inflammatory response in host brain via two 
different mechanisms, contact-dependent and contact-independent mechanisms $[3,7,8]$. The contact-dependent mechanism leads to direct host cell damage via active trogocytosis by $N$. fowleri trophozoites, in which food-cup structure (amoebastome) is involved. In the contact-independent mechanism, diverse cytolytic molecules released from the amoeba such as phospholipases, sphingomyelinase, elastase, neuroaminidase, and proteolytic enzymes may induce disruption and apoptosis of host cells in infected loci [3,9,10]. Studies investigating the biological properties and pathogenesis of $N$. fowleri have yet to fully elucidate the pathophysiology of the amoeba, which is necessary to widen our knowledge of the disease mechanisms and the nature of the amoeba.

In this study, we identified a novel cysteine protease inhibitor of $N$. fowleri (NfCPI) by data mining of the $N$. fowleri genomic resource and characterized its biochemical properties. NfCPI shows a dimeric structure and shares biochemical properties with proteins belonging to the cystatin family. Interestingly, the expression of NfCPI is increased during the encystation process and reaches a maximum in the mature cysts of $N$. fowleri, suggesting its essential role in regulation of encystation and formation of mature amoebic cysts.

\section{Materials and Methods}

\subsection{Cultivation of Naegleria fowleri}

Naegleria fowleri trophozoites (Carter NF69 strain, ATCC 30215) were cultured in Nelson medium supplemented with $2 \%$ heat-inactivated fetal bovine serum (FBS; Gibco, Rockville, MA, USA) and $1 \%$ penicillin-streptomycin (Gibco) at $37^{\circ} \mathrm{C}$ [11]. The amoeba were maintained by subculturing every three days and used in this study.

\subsection{Isolation and Cloning of a Gene Encoding NfCPI}

The gene encoding NfCPI (Gene ID: NF0117700) was identified by data mining the N. fowleri genomic resource (AmoebaDB, http://amoebadb.org/amoeba/, accessed on 16 December 2016). Total RNA of $N$. fowleri was purified using TRIzol reagent (Invitrogen, Carlsbad, CA, USA) and the purified total RNA was treated with RNase-free DNase (Gibco) to digest any contaminating DNA. The cDNA was synthesized from the RNA using the RNA to cDNA EcoDry Premix (Clontech, Mountain View, CA, USA) following the manufacturer's protocols. The $n f c p i$ was amplified using specific primers: 5'-ATGATCAACAACCTTTCCGCACCAGCA-3' and 5'-TCAAGCAGGTGTATTACAATCCACTCG-3'. Amplification was performed with a thermal parameter of $95^{\circ} \mathrm{C}$ for $10 \mathrm{~min}$; 25 cycles at $95^{\circ} \mathrm{C}$ for $1 \mathrm{~min}, 52^{\circ} \mathrm{C}$ for $1 \mathrm{~min}$, and $72{ }^{\circ} \mathrm{C}$ for $1 \mathrm{~min}$; and a final extension at $72{ }^{\circ} \mathrm{C}$ for $10 \mathrm{~min}$. The PCR product was purified, ligated into T\&A cloning vector (Real Biotech Corporation, Banqiao, Taiwan) and transformed into Escherichia coli DH5 $\alpha$ competent cells. The positive clones were selected by colony PCR, and the nucleotide sequences of the insert were confirmed by automatic DNA sequencing. Analysis of the primary structure of deduced amino acid sequences was performed with the DNASTAR package (DNASTAR, Madison, WI, USA). The signal peptide sequence and potential $n$-glycosylation sites were identified by SignalP5.0 (http:/ / www.cbs.dtu.dk/services/SignalP/, accessed on 21 January 2020) and netNGlyc 1.0 (http:/ / www.cbs.dtu.dk/services/NetNGlyc/, accessed on 21 January 2020), respectively. The phylogenetic tree was constructed with MEGA6 (http:/ / www.megasoftware.net, accessed on 2 July 2020) using the Maximum Likelihood Estimation (MLE) with Jones-Taylor-Thornton model. The robustness of the nodes was assessed with 1000 bootstrap replications.

\subsection{Expression and Purification of Recombinant NfCPI}

A partial gene of $n f c p i$ lacking the signal peptide sequence was amplified with the following primers: 5'-GGATCCCTTCCCATTGCAACCAATGTC-3' , containing a 5' BamHI site, and 5'-AAGCTTTCAAGCAGGTGTATTACAATC-3', containing a $5^{\prime}$ HindIII site. The amplified product was purified from the gel, and cloned into a T\&A vector (Real Biotech Corporation) as described above. The resulting plasmid DNA was digested with BamHI and HindIII, cloned into the corresponding restriction sites of pQE-9 expression vector 
(Qiagen, Valencia, CA, USA), and transformed into E. coli M15 (pREP14) competent cells (Qiagen). The selected E. coli clone was cultured in Luria Bertani broth, and expression of the NfCPI was induced with $1 \mathrm{mM}$ isopropyl-1-thio- $\beta$-D-galactopyranoside (IPTG) at $37^{\circ} \mathrm{C}$ for $4 \mathrm{~h}$. The cells were collected by centrifugation, suspended in native lysis buffer ( $50 \mathrm{mM}$ $\mathrm{NaH}_{2} \mathrm{PO}_{4}, 300 \mathrm{mM} \mathrm{NaCl}, 10 \mathrm{mM}$ imidazole, $\mathrm{pH}$ 8.0), sonicated on ice for $15 \mathrm{~min}$, and centrifuged at $4{ }^{\circ} \mathrm{C}$ for $30 \mathrm{~min}$ at $12,000 \mathrm{rpm}$. The supernatant was collected and the NfCPI was purified using the nickel-nitrilotriacetic acid (Ni-NTA) column (Qiagen) following the manufacturer's instructions. The purification and purity of the NfCPI were analyzed via $15 \%$ sodium dodecyl sulfate-polyacrylamide gel electrophoresis (SDS-PAGE) at $150 \mathrm{~V}$ for $1 \mathrm{~h}$.

\subsection{Inhibitory Activity Assay}

Inhibitory activity of NfCPI against cysteine proteases was determined by measuring the residual enzyme activity after incubation of each enzyme with NfCPI [12,13]. Cysteine proteases used in this study were papain (Sigma, St. Louis, MO, USA) and two recombinant cathepsin-B and cathepsin-B-like cysteine proteases of $N$. fowleri (NfCB and NfCBL). Recombinant NfCB and NfCBL were produced as described previously [9]. Briefly, $10 \mathrm{nM}$ of each enzyme was incubated with different concentrations of NfCPI (0-100 nM) in $50 \mathrm{mM}$ of sodium acetate $(\mathrm{pH} \mathrm{6.0)}$ for $20 \mathrm{~min}$ at room temperature (RT). Substrate solution was then added to the mixture, and the residual enzyme activity was determined by measuring the release of fluorescence (excitation at $355 \mathrm{~nm}$ and emission at $460 \mathrm{~nm}$ ) for 30 min at RT with a Fluoroskan Ascent FL (Thermo Fisher Scientific, Vantaa, Finland). Benzyloxycarbonyl-L-leucyl-L-arginine 4-methyl-coumaryl-7-amide (Z-LR-MCA; Peptide International, Louisville, KY, USA) was used as the substrate. Substrate solution was composed of $50 \mathrm{mM}$ sodium acetate ( $\mathrm{pH}$ 5.0), $10 \mathrm{nM}$ Z-LR-MCA, and $10 \mathrm{mM}$ dithiothreitol (DTT). The concentration of each cysteine protease was determined via active site titration with trans-epoxy-succinyl-L-leucylamido (4-guanidino)butane (E-64; Sigma). The concentration of NfCPI was measured via titration with papain as described previously [14].

\subsection{Biochemical Properties of NfCPI}

Effect of $\mathrm{pH}$ on the inhibitory activity of NfCPI was determined by incubating NfCPI $(20 \mathrm{nM})$ with the same concentration of papain, NfCB, or NfCBL in different $\mathrm{pH}$ buffers [50 mM sodium acetate ( $\mathrm{pH} 4.0-5.5), 50 \mathrm{mM}$ sodium phosphate ( $\mathrm{pH} \mathrm{6.0-6.5),} \mathrm{or} 50 \mathrm{mM}$ Tris- $\mathrm{HCl}$ (pH 7.0-8.0)] for $20 \mathrm{~min}$ at RT followed by an analysis of the residual activity of each enzyme using the same assay method described above. To determine $\mathrm{pH}$ stability of $\mathrm{NfCPI}$, the protein was incubated in different $\mathrm{pH}$ buffers at $37^{\circ} \mathrm{C}$ for $3 \mathrm{~h}$. The inhibitory activity of NfCPI preincubated under different $\mathrm{pH}$ conditions was assayed for papain, NfCB, or NfCBL. The thermal stability of NfCPI was determined by incubating NfCPI at different temperatures $\left(20,37\right.$, and $\left.95^{\circ} \mathrm{C}\right)$ for $0-3 \mathrm{~h}$ in $50 \mathrm{mM}$ sodium acetate ( $\mathrm{pH}$ 5.0). The samples were cooled on ice, and the residual inhibitory activities of sample aliquots against each cysteine protease were determined as described above. In all assays, E-64 was used as a control inhibitor. All the assays were conducted in triplicate, and the mean and standard deviation (SD) were calculated. The equilibrium dissociation constant $\left(K_{i}\right)$ values were calculated using the equation $K_{i}=\mathrm{IC}_{50}\left(1 /(1+[\mathrm{S}]) / K_{\mathrm{m}}\right)$ as described previously [15]. The $K_{\mathrm{m}}$ of each cysteine protease for the substrate was determined under the same assay conditions in the absence of NfCPI. The $K_{i}$ values were analyzed via non-linear regression using GraphPad Prism 8 Software (San Diego, CA, USA).

\subsection{Structural Analysis of NfCPI}

In order to analyze the structural properties of NfCPI, electrophoretic analysis of the NfCPI was performed in the presence and absence of SDS or $\beta$-mercaptoethanol [ $\beta-\mathrm{ME}, 5 \%$ (vol/vol)] with or without prior heating at $100{ }^{\circ} \mathrm{C}$ for $5 \mathrm{~min}[12,13]$. The native molecular size of NfCPI was also analyzed by gel filtration chromatography using a Superdex $200 \mathrm{HR}$ 10/30 column with an Äcta FPLC system (GE Biosciences, Pittsburgh, PA, USA). The 
purified NfCPI (1 mg) was loaded onto the column, and the collected fractions $(0.5 \mathrm{~mL})$ were analyzed by SDS-PAGE, followed by measurement of their inhibitory activities against NfCB and NfCBL. The column was calibrated with gel filtration size marker proteins (Sigma): blue dextran (2000 kDa), $\beta$-amylase (200 kDa), alcohol dehydrogenase (150 kDa), albumin $(66 \mathrm{kDa})$, carbonic anhydrase $(29 \mathrm{kDa})$, and cytochrome c $(12.4 \mathrm{kDa})$. The $K_{a v}$ value of each size marker protein was calculated using the equation $K_{a v}=\left(V_{e}-V_{0}\right) /\left(V_{t}-V_{0}\right)$, where $V_{e}$ is the elution volume of protein, $V_{0}$ refers to the elution volume of blue dextran, and $V_{t}$ is the total bed volume.

\subsection{Semiquantitative RT-PCR}

To determine the expression profiles of $n f c p i$ at different developmental stages of $N$. fowleri, semiquantitative RT-PCR was performed. Encystation of the amoeba was induced by incubating the trophozoites in encystation buffer $(120 \mathrm{mM} \mathrm{NaCl}, 0.03 \mathrm{mM} \mathrm{MgCl} 4,1 \mathrm{mM}$ $\mathrm{Na}_{2} \mathrm{HPO}_{4}, 1 \mathrm{mM} \mathrm{KH}_{2} \mathrm{PO}_{4}, 0.03 \mathrm{mM} \mathrm{CaCl}_{2}, 0.02 \mathrm{mM} \mathrm{FeCl}_{2}, \mathrm{pH}$ 6.8) [16] for $60 \mathrm{~h}$ at $37^{\circ} \mathrm{C}$. The morphological changes of the amoeba were analyzed with $\mathrm{EVOS}^{\circledR} \mathrm{XL}$ Core microscope (Life technologies, Carlsbad, CA, USA) and the cells were harvested every $12 \mathrm{~h}$. Total RNA was isolated from $N$. fowleri harvested at each time point using TRIzol (Invitrogen). The cDNA was synthesized using RNA to cDNA EcoDry Premix (Clontech). The transcriptional pattern of $n f c p i$ at each developmental stage was analyzed via semiquantitative RT-PCR. The expression profiles of $n f c b, n f c b l$, cathepsin Z $(n f c z)$ and calpain-like ( $n f c a l p a i n-l i k e)$ were also analyzed with the following primer sets: $n f c b\left(5^{\prime}\right.$-GAGC-TCATGATGATGAGATGCGTGAAT$3^{\prime}$ and $5^{\prime}$-AAGCTTTTAACAACATGCTCCATCA-ATGAAAT- $\left.3^{\prime}\right), n f c b l\left(5^{\prime}\right.$-GTGAGTAGGAGGTAGCCTTCCA- $3^{\prime}$ and $5^{\prime}$-CCTCTTGTTCA- TCACCTTGTTG-3'), $n f c z\left(5^{\prime}\right.$-ATGAAAAATCTCTGGCTTGTCTTGCTG-3 ${ }^{\prime}$ and $5^{\prime}$-TTAA-ACAATTGGAACAGCAAAGGCACA$3^{\prime}$ ), and nfcalpain-like (5'-ATGAACATTCCAGACAAAATTCCG-3' and 5'-TTACACAACTTTTCTAACTTC-3'). Glyceraldehyde 3-phosphate dehydrogenase of N. fowleri (nfgapdh) was included as an internal control for relative quantitation of other genes [13].

\subsection{Production of Monoclonal Antibody}

Monoclonal antibody (MAb) specific to NfCPI was produced according to the protocols described previously [17]. The purified NfCPI $(50 \mu \mathrm{g} / 100 \mu \mathrm{L})$ was mixed with an equal volume of Freund's complete adjuvant (Sigma), and the mixture was injected intraperitoneally (IP) into two six-week-old female BALB/c mice. The mice were boosted twice biweekly via IP injection of the NfCPI $(25 \mu \mathrm{g} / 100 \mu \mathrm{L})$ mixed with an equal volume of Freund's incomplete adjuvant (Sigma). After the third injection, the NfCPI $(5 \mu \mathrm{g} / 100 \mu \mathrm{L})$ was injected into mice tail intravenously (IV) without any adjuvant. The production of anti-NfCPI antibodies in immunized mice was confirmed by enzyme-linked immunosorbent assay (ELISA) with the NfCPI antigen $(1 \mu \mathrm{g} / \mathrm{mL})$. Goat anti-mouse immunoglobulin (Ig) G (Sigma, 1:5000 dilution) conjugated with alkaline phosphate was used as the secondary antibody and reactions were developed with a substrate buffer containing a p-nitrophenylphosphate (Sigma). Myeloma cells were fused with spleen cells from the immunized mice at a ratio of $1: 5$ by slowly adding $50 \%$ polyethyleneglycol (Sigma). The fused cells were selected with hypoxanthine, aminopterin, and thymidine (HAT) and HT media for two weeks. The supernatant of the hybridoma cells was screened by ELISA as described above. The selected hybridoma cells were cloned by limiting dilution, and the finally selected hybridoma colonies were transferred into $75 \mathrm{~cm}^{2}$ tissue culture flasks for mass culture. The supernatant was collected, concentrated, and used for further studies. All the protocols used in the mouse experiments were reviewed and approved by the Animal Research Ethics Committee of the Ajou University School of Medicine (AJ-IBC-17-09-10).

\subsection{Immunoblot}

Expression profile of NfCPI in the N. fowleri trophozoite and cyst was analyzed by immunoblotting. The lysates of $N$. fowleri trophozoites and cysts were prepared by repeated freezing and thawing procedures in RIPA Lysis and Extraction buffer (Thermo 
Fisher Scientific) followed by sonication on ice. The lysates of $N$. fowleri trophozoites $(20 \mu \mathrm{g})$ and cysts $(20 \mu \mathrm{g})$ were separated by 15\% SDS-PAGE and transferred electrophoretically onto the nitrocellulose membrane. The membrane was blocked with PBS supplemented with $0.05 \%$ Tween 20 (PBST) and 5\% skim milk for $1 \mathrm{~h}$ and incubated with anti-NfCPI MAb (1:1000 dilution in 5\% skim milk) or IgG purified from non-immunized mouse serum at RT for $2 \mathrm{~h}$. After several washes with PBST, the membrane was incubated with horseradish peroxidase (HRP)-conjugated anti-mouse IgG (Sigma, 1:1000 dilution in 5\% skim milk) at RT for $2 \mathrm{~h}$. The membrane was washed with PBST several times and immunoreactive bands were visualized with 4-chloro-1-naphthol (Sigma).

\subsection{Immunofluorescence Assay (IFA)}

Localization of NfCPI at different developmental stages of $N$. fowleri was determined by IFA with a slightly modified method [18]. N. fowleri trophozoites and cysts on the coverslip were fixed with $10 \%$ formaldehyde for $10 \mathrm{~min}$ at room temperature, permeabilized in $1 \%$ ammonium hydroxide, washed in Tween 20 for $5 \mathrm{~min}$, and then washed extensively with $0.82 \%$ physiological saline. Anti-NfCPI MAb (1:100 diluted in PBS) was added to the trophozoites and cysts and incubated overnight at $4{ }^{\circ} \mathrm{C}$. After washing with PBS several times, fluorescent isothiocyanate (FITC)-conjugated goat anti-mouse IgG (Sigma, 1:200 dilution) was added and incubated at $4{ }^{\circ} \mathrm{C}$ for $2 \mathrm{~h}$. Fluorescence was visualized in the optical section produced by confocal laser scanning microscopy (Carl Zeiss, Oberkochen, Germany).

\subsection{Statistical Analysis}

All experiments were performed in triplicate. Statistical significance was evaluated by one-way ANOVA, followed by one-tailed Student's $t$ test. Differences in mean values at $p<0.05$ were considered statistically significant.

\section{Results}

\subsection{Sequence Analysis of NfCPI}

The open reading frame of $n f c p i$ consisted of $411 \mathrm{bp}$ encoding 136 amino acid residues with a predicted molecular mass of $14.7 \mathrm{kDa}$. Analysis of deduced amino acid sequence revealed that NfCPI had a typical N-terminal signal peptide sequence of 29 amino acids (MINNLSAPAAATTLIFILALLITFSNILA) and two potential $N$-glycosylation sites at 4NLS6 and 46NQT48. Multiple sequence alignment of amino acid sequences of NfCPI and related proteins from other organisms revealed low sequence identity. However, the Gln-Val-ValAla-Gly (QVVAG) motif, a tightly conserved motif of cystatin family, was identified in NfCPI (Figure 1A). Two C-terminal cysteine residues that form a disulfide bond in the cystatin C, D, and S superfamily $[19,20]$ were also detected in the NfCPI. Phylogenetic analysis of NfCPI with other related proteins also revealed that NfCPI formed a large cluster with cystatin C, D, and S superfamily proteins (Figure 1B).

\subsection{Expression and Purification of NfCPI}

A fragment of $n f c p i$ lacking the $29 \mathrm{~N}$-terminal amino acid sequences that correspond to signal peptide sequence was expressed in E. coli. The NfCPI was expressed as a soluble protein with an approximate molecular mass of $12 \mathrm{kDa}$, which coincided with the expected molecular mass of the protein calculated with the deduced amino acid sequences. The recombinant protein was purified using Ni-NTA affinity chromatography (Figure 2A).

\subsection{Dimeric Structure of NfCPI}

Electrophoretic analysis of NfCPI under non-reducing conditions revealed that the protein formed a dimeric structure. The size of NfCPI in non-reducing PAGE was approximately $24 \mathrm{kDa}$ (Figure 2B). The size was not affected by SDS. Meanwhile, $\beta$-ME affected the electrophoretic movement of NfCPI to a size of about $12 \mathrm{kDa}$ in the presence or absence of heating, suggesting the role of inter-disulfide bonds in the dimeric structure formation of NfCPI. To further analyze the native size of NfCPI, gel filtration chromatography was 
performed. The NfCPI was mainly detected in the fractions 26 to 28, which corresponded to the predicted molecular size of $24 \mathrm{kDa}$ (Figure 2C).
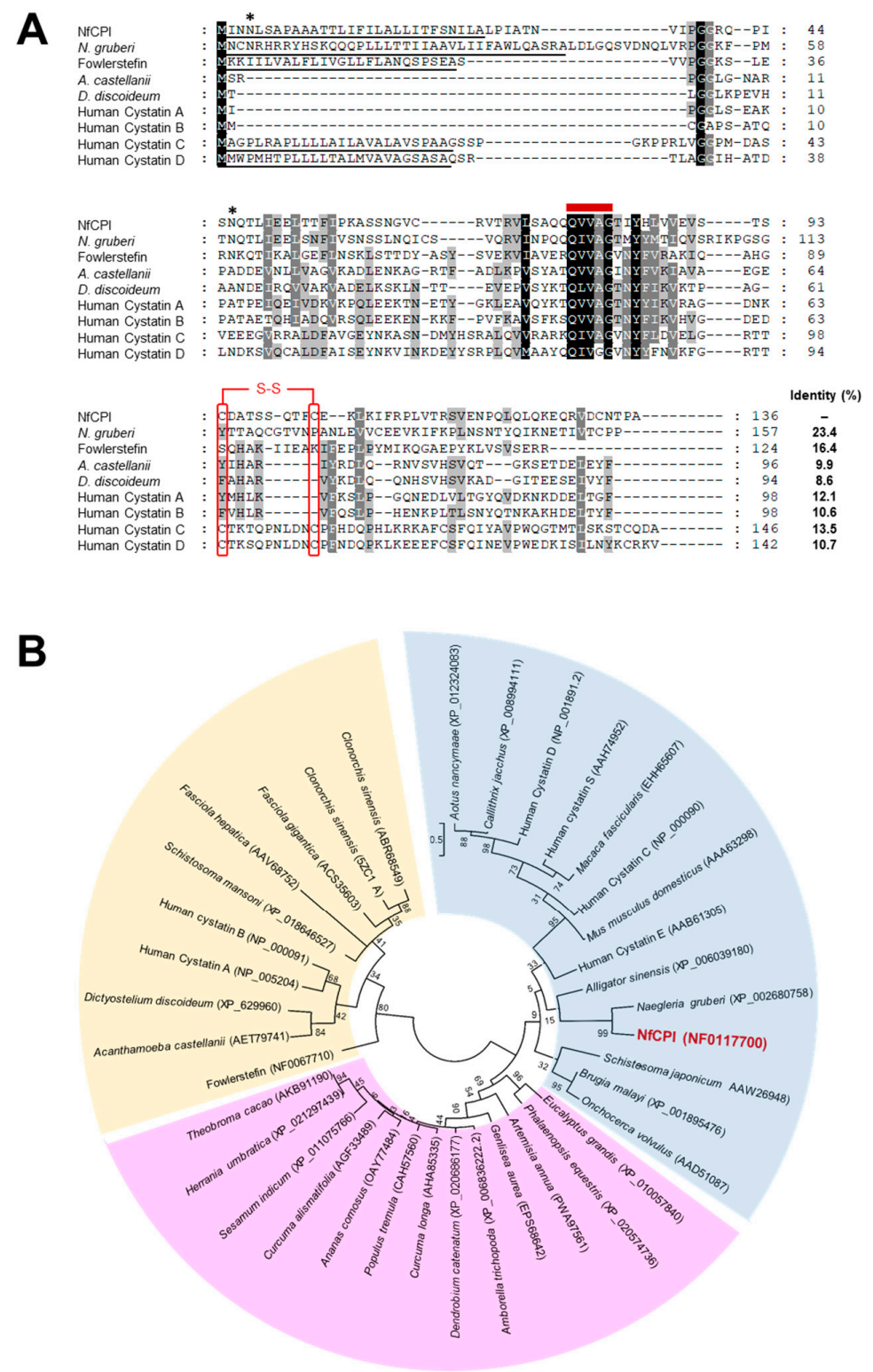

Figure 1. Multiple sequence alignment and phylogenetic analysis. (A) Multiple sequence alignment. The deduced amino acid sequence of $N$. fowleri (NfCPI) was aligned with the sequences of related proteins derived from other protozoa and humans. The Gln-Val-Val-Ala-Gly (QVVAG) cystatin motif is presented as a bold red line on the sequences. The predicted $\mathrm{N}$-terminal signal peptide sequences are underlined. The predicted putative $\mathrm{N}$-glycosylation sites in NfCPI are marked by asterisks. A potential disulfide bridge is presented in brackets and the associated cysteine residues are boxed in red color. The shading displays the degree of identity among the sequences. Sequence identity between NfCPI and other related proteins is shown on the right side. Sequence identity between NfCPI and other related proteins is shown on the right side. Naegleria gruberi CPI (XP_002680758.1), Fowlerstefin (NF0067710), Acanthamoeba castellanii CPI (AET79741), Dictyostelium discoideum CPI (XP_629960), Human cystatin A (NP_005204), Human cystatin B (NM_000100), Human cystatin C (NP_000090), and Human cystatin D (NP_001891) are included in the alignment. (B) The phylogenetic tree was constructed by the maximum likelihood method using the MEGA6 program. Yellow, cystatin A and B clades; blue, cystatin C, D, and S clades; pink, CPIs from plants. Numbers on the branches show bootstrap proportion (1000 replicates). 

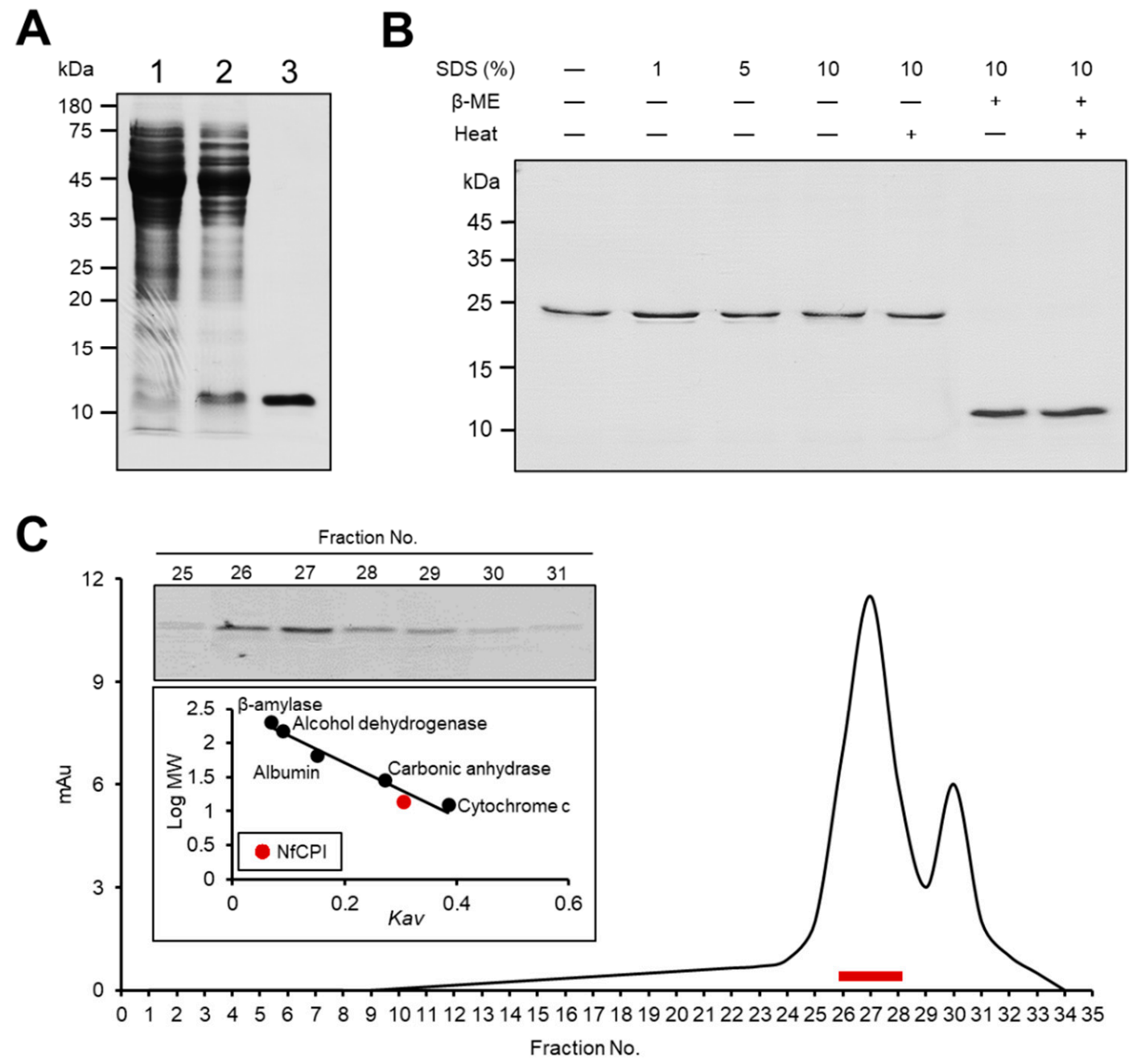

Figure 2. Expression of recombinant NfCPI and structural analysis. (A) The NfCPI was purified using Ni-NTA affinity chromatography and analyzed via sodium dodecyl sulfate-polyacrylamide gel electrophoresis (SDS-PAGE). Lane 1, noninduced E. coli lysate; lane 2, isopropyl-1-thio- $\beta$-D-galactopyranoside (IPTG)-induced E. coli lysate; lane 3, Ni-NTA affinity purified NfCPI. (B) The NfCPI was mixed with different concentrations of SDS $(0-10 \%, v / v)$ and $\beta$-ME with or without heating and analyzed via SDS-PAGE. (C) Gel filtration chromatography analysis. The NfCPI was loaded to Superdex 200 HR 10/30 column and fractions $(0.5 \mathrm{~mL})$ were collected. The collected fractions were analyzed by SDS-PAGE and their inhibitory activities against NfCB and NfCBL were assayed. The fractions expressing strong inhibitory activity (fractions 26 to 28 ) are represented as red bars and confirmed by SDS-PAGE. The $K_{a v}$ value of NfCPI (red dot) was calculated by comparing with those of size markers (black dots).

\subsection{Inhibitory Activity of NfCPI}

Inhibitory activity of NfCPI against several cysteine proteases including papain, NfCB, and NfCBL was analyzed. NfCPI inhibited the enzyme activities of all the tested enzymes in a dose-dependent manner. However, it showed greater inhibition against NfCB and NfCBL, endogenous cysteine proteases of $N$. fowleri, than papain (Figure $3 \mathrm{~A})$. The $K_{i}$ values also suggested that NfCPI inhibited NfCB and NfCBL more effectively than papain in the picomolar range (Table 1). NfCPI inhibited the tested enzymes under a broad range of $\mathrm{pH}$ values, suggesting that the interactions between NfCPI and the enzymes were not influenced by $\mathrm{pH}$ (Figure 3B). NfCPI was stable under different $\mathrm{pH}$ conditions resulting in no significant change in its inhibitory capacity (Figure 3C). The thermal stability analysis revealed the heat-labile features of NfCPI. The protein was stable at 20 and $37^{\circ} \mathrm{C}$ at least for $3 \mathrm{~h}$, but it rapidly lost its inhibitory activity when incubated at $95^{\circ} \mathrm{C}$ (Figure $3 \mathrm{D}$ ). 

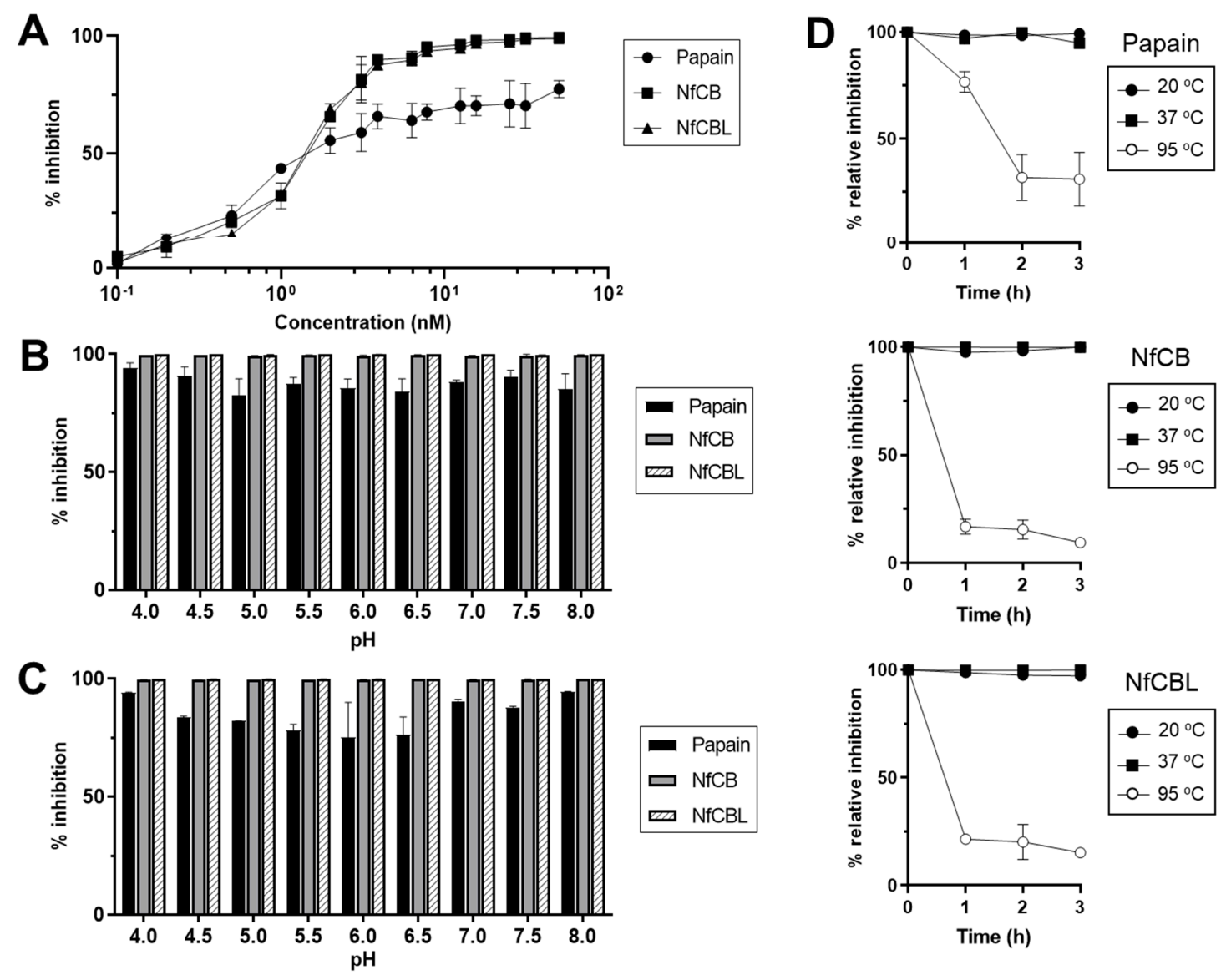

Figure 3. Inhibitory activity of NfCPI. (A) Inhibitory activity of NfCPI against cysteine proteases. Different concentrations of NfCPI (0-100 nM) were incubated with each cysteine protease $(10 \mathrm{nM})$ and the residual enzyme activity of each enzyme was assayed. (B) pH dependency. NfCPI was incubated with papain, NfCB and NfCBL in different pH buffers and the residual enzyme activity was analyzed. (C) $\mathrm{pH}$ stability. NfCPI was incubated in different $\mathrm{pH}$ buffers for $3 \mathrm{~h}$ and its inhibitory activity against cysteine proteases was assayed. (D) Thermal stability. NfCPI was incubated at 20,37 , and $95{ }^{\circ} \mathrm{C}$ for the indicated time points, respectively. The inhibitory activity of NfCPI against cysteine proteases was analyzed. All experiments were performed in triplicate and the mean and standard deviation (SD) values are presented.

Table 1. $K_{i}$ values of NfCPI against cysteine proteases.

\begin{tabular}{cc}
\hline Enzyme & $\boldsymbol{K}_{\boldsymbol{i}}(\mathbf{n M}) \pm \mathrm{SD}$ \\
\hline Papain & $0.259 \pm 0.034$ \\
NfCB & $0.193 \pm 0.032$ \\
NfCBL & $0.144 \pm 0.003$ \\
\hline
\end{tabular}

\subsection{Expression Pattern and Cellular Localization of NfCPI}

The trophozoites of $N$. fowleri were successfully transformed to cysts following incubation at $60 \mathrm{~h}$ in the encystation medium (Figure 4A). RT-PCR was performed using the RNAs isolated at each stage during the encystation process to investigate the transcription profiles of $n f c p i$ from trophozoites to cysts. The expression of $n f c p i$ was not detectable at the trophozoite stage, but it gradually increased starting at $12 \mathrm{~h}$ post-incubation, suggesting that $n f c p i$ was mainly expressed during encystation and in mature cysts (Figure 4B). Expression profiles of several endogenous cysteine proteases, including $n f c b, n f c b l, n f c z$ and $n f c a l p a i n-l i k e$, were also analyzed. For $n f c b, n f c b l$, and $n f c z$, the highest levels of expression were identified in trophozoites and the gene expression level declined eventually as the trophozoites transformed into cysts. Meanwhile, nfcalpain-like was not expressed in trophozoites. However, its expression was gradually increased at $12 \mathrm{~h}$ post-incubation, which 
coincided with the expression pattern of $n f c p i$. In order to confirm the expression profile of NfCPI at different developmental stages, an immunoblot using lysates of $N$. fowleri trophozoites and cysts and anti-NfCPI MAb was performed. Immunoblot results revealed elevated levels of NfCPI in cysts but not in trophozoites (Figure 4C). IFA also revealed that NfCPI was mainly identified in the cytosol of cysts in clusters that were not further identified (Figure 4D).
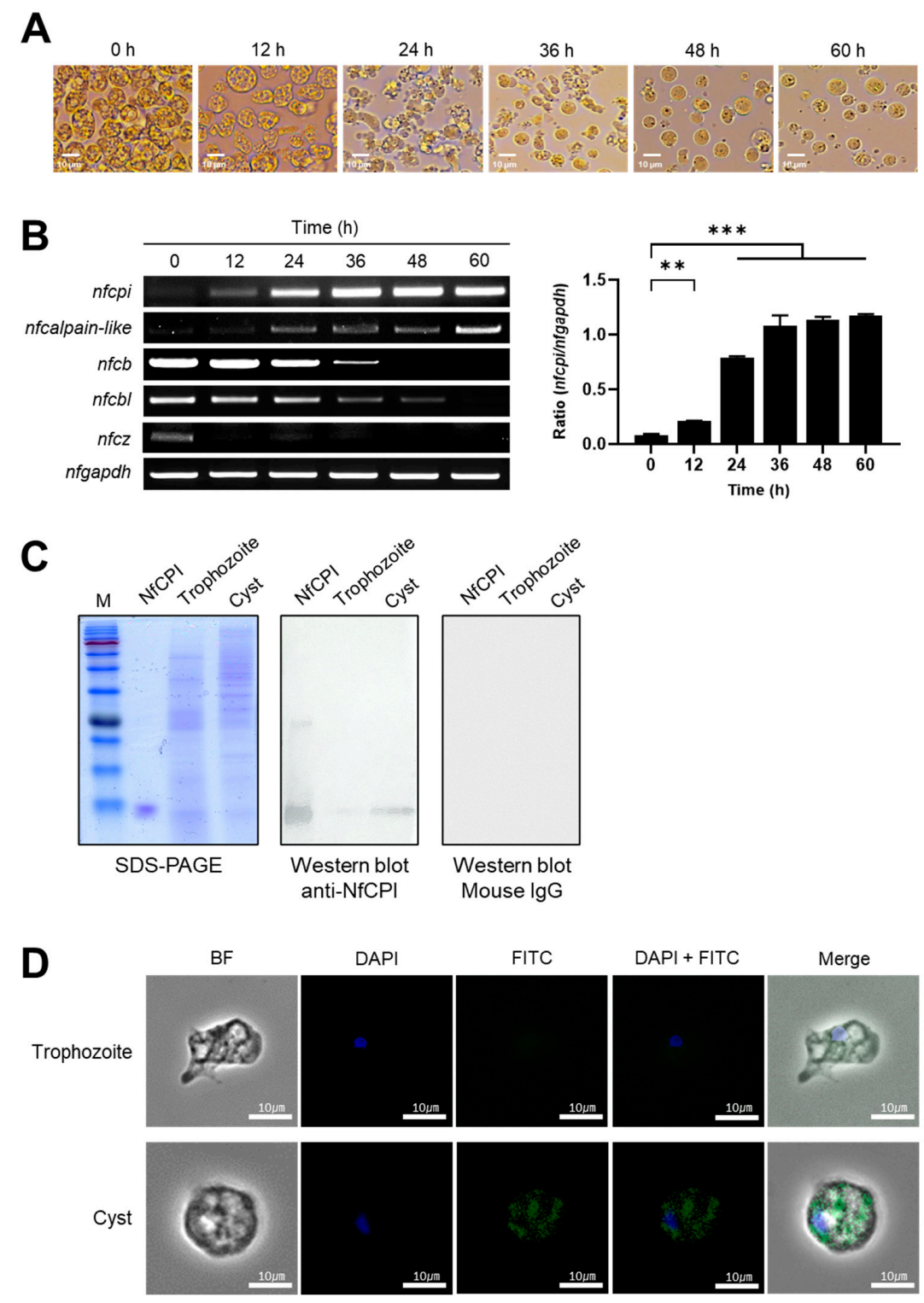

Figure 4. Expression pattern of NfCPI in N. fowleri. (A) The morphological changes of N. fowleri during encystation. N. fowleri trophozoites were incubated in encystation medium and morphological changes of the amoeba were analyzed at the indicated time points by microscopy. (B) Semiquantitative RT-PCR. The transcription profiles of $n f c p i, n f c b, n f c b l, n f c z$, and $n f c a l p a i n-l i k e$ were analyzed during encystation (left panel). Graphs show the mean \pm SD densitometric ratios of $n f c p i$ and nfgapdh of three independent experiments (right panel). Significance of the data was analyzed by using one-tailed $t$ test, ${ }^{* *} p<0.005,{ }^{* * *} p<0.001$. (C) Immunoblot analysis. Expression profile of NfCPI in trophozoites and cysts was determined by immunoblot with anti-NfCPI MAb or non-immunized mouse IgG. (D) Localization. Immunofluorescence Assay (IFA) was performed with anti-NfCPI MAb. N. fowleri trophozoites and cysts were fixed on cover slip and probed with anti-NfCPI MAb and FITC-conjugated anti-mouse IgG. The slide was observed with a confocal laser scanning microscope. 


\section{Discussion}

Proteases of protozoan parasites play essential biological functions in the pathophysiology and pathobiology of organisms [21]. Several classes of proteases of N. fowleri have attracted great attention because of their key roles in the physiology and pathogenesis of amoeba $[9,22-26]$. Although these enzymes play pivotal roles in physiology and pathobiology of amoeba, a stringent regulation of their activities is also essential to control or modulate normal biological processes in amoeba. Studies have reported the essential role of parasitic protozoan cysteine protease inhibitors in the survival and infectivity of parasites in hosts by regulating endogenous or exogenous proteases [27-30]. Previously, we identified and characterized a novel stefin-family cysteine protease inhibitor of $N$. fowleri, known as fowlerstefin [13]. In this study, we characterized the second cysteine protease inhibitor of $N$. fowleri, NfCPI.

$\mathrm{NfCPI}$ is a typical cysteine protease inhibitor belonging to the superfamily of cystatin $\mathrm{C}$, D, and $\mathrm{S}$ superfamily proteins. The QVVAG motif, which forms a hairpin loop involved in direct interaction with target enzymes [19,31],was conserved in NfCPI. Two C-terminal cysteine residues that may form disulfide bonds were also found in NfCPI. Non-reducing PAGE and gel infiltration analyses suggested a dimeric structure of NfCPI, which further support the involvement of the two cysteine residues in inter-disulfide bridge formation. Dimeric structures linked by inter-disulfide bonds are usually identified in extracellular cystatins $[19,20]$. Although NfCPI has a potential signal peptide sequence at its $\mathrm{N}$-terminal region, it is not clear whether it is actively secreted outside of $N$. fowleri because the protein was mainly detected in the cytoplasmic region of the cysts.

$\mathrm{NfCPI}$ effectively inhibited cysteine proteases such as $\mathrm{NfCB}, \mathrm{NfCBL}$, and papain under a broad range of $\mathrm{pH}$ conditions, suggesting that $\mathrm{NfCPI}$ is a functional protein. Although $\mathrm{NfCPI}$ effectively inhibited two endogenous cysteine proteases of N. fowleri, NfCB and $\mathrm{NfCBL}$, it is likely that the two enzymes may not represent the primary targets of NfCPI since the transcription patterns of the two counterpart proteins did not coincide with that of $n f c p i$. The transcription levels of $n f c b$ and $n f c b l$ were the highest in trophozoites and decreased significantly during the encystations of the amoeba. In contrast, the transcriptional level of $n f c p i$ was gradually increased in the amoeba during encystation and reached a maximum in the mature cysts. Immunoblot and IFA results also supported its high level of expression in cysts. Data mining of the N. fowleri genomic resource (AmoebaDB) shed light on diverse cysteine proteases of the amoeba. We analyzed stage-specific transcriptional patterns of the genes encoding candidate cysteine proteases of N. fowleri and found that transcription profile of Nfcalpain-like enzyme (nfcalpain-like) was identical to that of $n f c p i$. The expression patterns of the other candidate enzymes including $n f c b, n f c b l$, other homologs of $n f c b$, and $n f c z$ were not consistent with the expression profile of $n f c p i$. These results suggested that Nfcalpain-like enzyme could be a potential primary target for NfCPI. Calpains are $\mathrm{Ca}^{2+}$-dependent cysteine proteases belonging to clan CA protease. They play essential roles in a range of crucial cellular processes in diverse organisms including humans, animals, plants, nematodes, and protozoa [32,33]. Although the calpain family of enzymes is well characterized in animals and plants, their role in unicellular eukaryotes including $N$. fowleri is unknown. We tried the expression of recombinant Nfcalpain-like enzyme to determine the functional relevance of NfCPI as a calpain inhibitor. However, unfortunately, we failed to obtain an enzymatically active recombinant Nfcalpain-like protein. Further studies are necessary to determine whether Nfcalpain-like is a genuine target of NfCPI.

The biological function of NfCPI is not clear. Given its increased expression in amoeba under encystation and that it reaches maximum levels in mature cysts, NfCPI may play an important role in encystation and cyst formation of N. fowleri. Encystation is an essential step in differentiation of amoeba during metabolic and transcriptional changes, which initiate or induce large modifications of subcellular organelles as well as morphological changes of the amoeba itself [34-36]. The functional significance of proteases in amoeba cyst formation has been partially determined in few previous studies. Entamoeba invadens 
EiCP-B is specifically expressed near the cyst wall during encystation [37]. A serine protease M17 leucine aminopeptidase of Acanthamoeba castellanii (AcLAP) plays an important role in mature cyst formation [38]. In Naegleria spp., the molecular mechanisms associated with encystation have not been clearly understood. The role of proteases in encystation of the amoeba is also unknown. Only a few molecules, including ubiquitin-like Atg8 and enolase, have been reported to be associated with encystation [39,40]. Given the specific expression of NfCPI during encystation of $N$. fowleri, it could play a critical role in regulating the proteolytic activities of cysteine proteases, mediating the encystation process or mature cyst formation in amoeba.

\section{Conclusions}

NfCPI is a functional cystatin-family protease inhibitor of N. fowleri. Specific expression of the protein during encystation and at mature cyst of the amoeba suggests its potential roles in encystation and cyst formation of N. fowleri. Given the highly limited knowledge of the molecular mechanism underlying the induction or modulation of encystation of $N$. fowleri, further studies exploring the role of proteases and protease inhibitors are necessary to obtain an in-depth insight into the molecular mechanism of encystation of the amoeba. Studies investigating the molecular mechanism of excystation of the amoeba will also contribute to our understanding of the biological properties and pathogenicity of the amoeba.

Author Contributions: Conceptualization, H.G.L. and B.-K.N.; Methodology, H.G.L. and A.-J.H.; Formal analysis, H.G.L., A.-J.H., J.-M.K., T.C.V., H.N. and H.-J.S. (Hae-Jin Sohn); Investigation, H.G.L. and B.-K.N.; Supervision, B.-K.N.; Project Administration, B.-K.N.; Funding Acquisition, B.-K.N.; Writing-original draft preparation, H.G.L. and B.-K.N.; Writing-review and editing, H.G.L., J.-M.K., H.-J.S. (Hae-Jin Sohn), H.-J.S. (Ho-Joon Shin), and B.-K.N. All authors have read and agreed to the published version of the manuscript.

Funding: This study was supported by the National Research Foundation of Korea (NRF) grant funded by the Korea Government (NRF-2018R1D1A1B07048097).

Institutional Review Board Statement: All the protocols used in the mouse experiments were reviewed and approved by the Animal Research Ethics Committee of the Ajou University School of Medicine, Korea (AJ-IBC-17-09-10).

Informed Consent Statement: Not applicable.

Data Availability Statement: The original data in the present study are available from the corresponding author upon request.

Conflicts of Interest: The authors declare no conflict of interest.

\section{References}

1. Yoder, J.S.; Eddy, B.A.; Visvesvara, G.S.; Capewell, L.; Beach, M.J. The epidemiology of primary amoebic meningoencephalitis in the USA, 1962-2008. Epidemiol. Infect. 2010, 138, 968-975. [CrossRef]

2. Visvesvara, G.S.; Moura, H.; Schuster, F.L. Pathogenic and opportunistic free-living amoebae: Acanthamoeba spp., Balamuthia mandrillaris, Naegleria fowleri, and Sappinia diploidea. FEMS Immunol. Med. Microbiol. 2007, 50, 1-26. [CrossRef]

3. Marciano-Cabral, F.; Cabral, G.A. The immune response to Naegleria fowleri amebae and pathogenesis of infection. FEMS Immunol. Med. Microbiol. 2007, 51, 243-259. [CrossRef] [PubMed]

4. Baig, A.M.; Khan, N.A. Novel chemotherapeutic strategies in the management of primary amoebic meningoencephalitis due to Naegleria fowleri. CNS Neurosci. Ther. 2014, 20, 289-290. [CrossRef] [PubMed]

5. Gharpure, R.; Gleason, M.; Salah, Z.; Blackstock, A.J.; Hess-Homeier, D.; Yoder, J.S.; Ali, I.K.M.; Collier, S.A.; Cope, J.R. Geographic range of recreational water-associated primary amebic meningoencephalitis, United States, 1978-2018. Emerg. Infect. Dis. 2021, 27, 271-274. [CrossRef]

6. Gharpure, R.; Bliton, J.; Goodman, A.; Ali, I.K.M.; Yoder, J.; Cope, J.R. Epidemiology and clinical characteristics of primary amebic meningoencephalitis caused by Naegleria fowleri: A Global Review. Clin. Infect. Dis. 2020, ciaa520. [CrossRef] [PubMed]

7. Grace, E.; Asbill, S.; Virga, K. Naegleria fowleri: Pathogenesis, diagnosis, and treatment options. Antimicrob. Agents Chemother. 2015, 59, 6677-6681. [CrossRef] [PubMed] 
8. Siddiqui, R.; Ali, I.K.M.; Cope, J.R.; Khan, N.A. Biology and pathogenesis of Naegleria fowleri. Acta Trop. 2016, $164,375-394$. [CrossRef] [PubMed]

9. Lee, J.; Kim, J.H.; Sohn, H.J.; Yang, H.J.; Na, B.K.; Chwae, Y.J.; Park, S.; Kim, K.; Shin, H.J. Novel cathepsin B and cathepsin B-like cysteine protease of Naegleria fowleri excretory-secretory proteins and their biochemical properties. Parasitol. Res. 2014, 113, 2765-2776. [CrossRef] [PubMed]

10. Fulford, D.E.; Marciano-cabral, F. Cytolytic Activity of Naegleria fowleri Cell-free Extract. J. Protozool. 1986, 33, 498-502. [CrossRef] [PubMed]

11. Nelson, E.C.; Jones, M. Culture isolation of agents of primary amebic meningcencephalitis. J. Parasitol. 1970, 56, $248-249$.

12. Kang, J.M.; Lee, K.H.; Sohn, W.M.; Na, B.K. Identification and functional characterization of CsStefin-1, a cysteine protease inhibitor of Clonorchis sinensis. Mol. Biochem. Parasitol. 2011, 177, 126-134. [CrossRef]

13. Thaí, T.L.; Kang, J.M.; Lê, H.G.; Lee, J.; Yoo, W.G.; Shin, H.J.; Sohn, W.M.; Na, B.K. Fowlerstefin, a cysteine protease inhibitor of Naegleria fowleri, induces inflammatory responses in BV-2 microglial cells in vitro. Parasites Vectors 2020, 13, 1-15. [CrossRef] [PubMed]

14. Monteiro, A.C.S.; Abrahamson, M.; Lima, A.P.C.A.; Vannier-Santos, M.A.; Scharfstein, J. Identification, characterization and localization of chagasin, a tight-binding cysteine protease inhibitor in Trypanosoma cruzi. J. Cell Sci. 2001, 114, 3933-3942.

15. Sanderson, S.J.; Westrop, G.D.; Scharfstein, J.; Mottram, J.C.; Coombs, G.H. Functional conservation of a natural cysteine peptidase inhibitor in protozoan and bacterial pathogens. FEBS Lett. 2003, 542, 12-16. [CrossRef]

16. Sohn, H.J.; Kang, H.; Seo, G.E.; Kim, J.H.; Jung, S.Y.; Shin, H.J. Efficient liquid media for encystation of pathogenic free-living amoebae. Korean J. Parasitol. 2017, 55, 233-238. [CrossRef]

17. Seong, G.S.; Sohn, H.J.; Kang, H.; Seo, G.E.; Kim, J.H.; Shin, H.J. Production and characterization of monoclonal antibodies against cathepsin B and cathepsin B-Like proteins of Naegleria fowleri. Exp. Parasitol. 2017, 183, 171-177. [CrossRef] [PubMed]

18. Sohn, H.J.; Song, K.J.; Kang, H.; Ham, A.J.; Lee, J.H.; Chwae, Y.J.; Kim, K.; Park, S.; Kim, J.H.; Shin, H.J. Cellular characterization of actin gene concerned with contact-dependent mechanisms in Naegleria fowleri. Parasite Immunol. 2019, 41, e12631. [CrossRef] [PubMed]

19. Turk, V.; Stoka, V.; Turk, D. Cystatins: Biochemical and structural properties, and medical relevance. Front. Biosci. 2008, 13, 5406-5420. [CrossRef]

20. Abrahamson, M.; Alvarez-Fernandez, M.; Nathanson, C.M. Cystatins. Biochem. Soc. Symp. 2003, 70, 179-199. [CrossRef]

21. McKerrow, J.H. The diverse roles of cysteine proteases in parasites and their suitability as drug targets. PLoS Negl. Trop. Dis. 2018, 12, e0005639. [CrossRef]

22. Serrano-Luna, J.; Cervantes-Sandoval, I.; Tsutsumi, V.; Shibayama, M. A biochemical comparison of proteases from pathogenic Naegleria fowleri and non-pathogenic Naegleria gruberi. J. Eukaryot. Microbiol. 2007, 54, 411-417. [CrossRef] [PubMed]

23. Lowrey, D.M.; McLaughlin, J. Activation of a heat-stable cytolytic protein associated with the surface membrane of Naegleria fowleri. Infect. Immun. 1985, 50, 478-482. [CrossRef] [PubMed]

24. Mat Amin, N. Proteinases in Naegleria fowleri (strain NF3), a pathogenic amoeba: A preliminary study. Trop. Biomed. 2004, 21, 57-60. [PubMed]

25. Vyas, I.K.; Jamerson, M.; Cabral, G.A.; Marciano-Cabral, F. Identification of peptidases in highly pathogenic vs. weakly pathogenic Naegleria fowleri amebae. J. Eukaryot. Microbiol. 2015, 62, 51-59. [CrossRef]

26. Aldape, K.; Huizinga, H.; Bouvier, J.; McKerrow, J. Naegleria fowleri: Characterization of a Secreted Histolytic Cysteine Protease. Exp. Parasitol. 1994, 78, 230-241. [CrossRef]

27. Liu, J.; Svärd, S.G.; Klotz, C. Giardia intestinalis cystatin is a potent inhibitor of papain, parasite cysteine proteases and, to a lesser extent, human cathepsin B. FEBS Lett. 2019, 593, 1313-1325. [CrossRef]

28. Pandey, K.C.; Singh, N.; Arastu-Kapur, S.; Bogyo, M.; Rosenthal, P.J. Falstatin, a cysteine protease inhibitor of Plasmodium falciparum, facilitates erythrocyte invasion. PLoS Pathog. 2006, 2, e117. [CrossRef]

29. Rennenberg, A.; Lehmann, C.; Heitmann, A.; Witt, T.; Hansen, G.; Nagarajan, K.; Deschermeier, C.; Turk, V.; Hilgenfeld, R.; Heussler, V.T. Exoerythrocytic Plasmodium parasites secrete a cysteine protease inhibitor involved in sporozoite invasion and capable of blocking cell death of host hepatocytes. PLoS Pathog. 2010, 6, e1000825. [CrossRef]

30. Kang, J.M.; Ju, H.L.; Yu, J.R.; Sohn, W.M.; Na, B.K. Cryptostatin, a chagasin-family cysteine protease inhibitor of Cryptosporidium parvum. Parasitology 2012, 139, 1029-1037. [CrossRef]

31. Stubbs, M.T.; Laber, B.; Bode, W.; Huber, R.; Jerala, R.; Lenarcic, B.; Turk, V. The refined $2.4 \AA$ X-ray crystal structure of recombinant human stefin B in complex with the cysteine proteinase papain: A novel type of proteinase inhibitor interaction. EMBO J. 1990, 9 , 1939-1947. [CrossRef]

32. Ono, Y.; Sorimachi, H. Calpains-An elaborate proteolytic system. Biochim. Biophys. Acta-Proteins Proteom. 2012, 1824, 224-236. [CrossRef] [PubMed]

33. Zhao, S.; Liang, Z.; Demko, V.; Wilson, R.; Johansen, W.; Olsen, O.A.; Shalchian-Tabrizi, K. Massive expansion of the calpain gene family in unicellular eukaryotes. BMC Evol. Biol. 2012, 12, 193. [CrossRef] [PubMed]

34. De Cádiz, A.E.; Jeelani, G.; Nakada-Tsukui, K.; Caler, E.; Nozaki, T. Transcriptome analysis of encystation in Entamoeba invadens. PLoS ONE 2013, 8, e74840. [CrossRef] [PubMed] 
35. Jeelani, G.; Sato, D.; Husain, A.; Escueta-de Cadiz, A.; Sugimoto, M.; Soga, T.; Suematsu, M.; Nozaki, T. Metabolic profiling of the protozoan parasite Entamoeba invadens revealed activation of unpredicted pathway during encystation. PLoS ONE 2012, 7, e37740. [CrossRef]

36. Moon, E.K.; Xuan, Y.H.; Chung, D.I.; Hong, Y.; Kong, H.H. Microarray analysis of differentially expressed genes between cysts and trophozoites of acanthamoeba castellanii. Korean J. Parasitol. 2011, 49, 341-347. [CrossRef]

37. Ebert, F.; Bachmann, A.; Nakada-Tsukui, K.; Hennings, I.; Drescher, B.; Nozaki, T.; Tannich, E.; Bruchhaus, I. An Entamoeba cysteine peptidase specifically expressed during encystation. Parasitol. Int. 2008, 57, 521-524. [CrossRef]

38. Lee, Y.R.; Na, B.K.; Moon, E.K.; Song, S.M.; Joo, S.Y.; Kong, H.H.; Goo, Y.K.; Chung, D.I.; Hong, Y. Essential role for an M17 leucine aminopeptidase in encystation of Acanthamoeba castellanii. PLoS ONE 2015, 10, e0129884. [CrossRef] [PubMed]

39. Cárdenas-Zúñiga, R.; Sánchez-Monroy, V.; Bermúdez-Cruz, R.M.; Rodríguez, M.A.; Serrano-Luna, J.; Shibayama, M. Ubiquitinlike Atg8 protein is expressed during autophagy and the encystation process in Naegleria gruberi. Parasitol. Res. 2017, 116, 303-312. [CrossRef]

40. Chávez-Munguía, B.; Segovia-Gamboa, N.; Salazar-Villatoro, L.; Omaña-Molina, M.; Espinosa-Cantellano, M.; MartÍnez-Palomo, A. Naegleria fowleri: Enolase is expressed during cyst differentiation. J. Eukaryot. Microbiol. 2011, 58, 463-468. [CrossRef] 